\title{
Stratum corneum lipid matrix with unusual packing: A molecular dynamics study
}

\author{
Egipto Antunes, Artur Cavaco-Paulo* \\ CEB - Centre of Biological Engineering, University of Minho, 4710-057 Braga, Portugal
}

\section{A R T I C L E I N F O}

\section{Keywords:}

Lipid matrix

Molecular dynamics simulations

Skin

Splayed ceramides

Stratum corneum

Sebum

\begin{abstract}
A B S T R A C T
The skin is an effective barrier against the external elements being the stratum corneum, with its lipid matrix surrounding the corneocytes, considered the major player responsible for its low permeability. The use of computational models to study the transdermal delivery of compounds have a huge potential to improve this research area, but requires reliable models of the skin components. In this work, we developed molecular dynamics models with a coarse-grained resolution, of the stratum corneum lipid matrix and the sebum. We developed the lipid matrix model with unusual lipid packing configuration as some recent works support. The simulation results show that this configuration is stable and may help to explain the low permeability of stratum corneum. The sebum simulations showed that this oily skin product can also play a significant role in the transdermal delivery of drugs.
\end{abstract}

\section{Introduction}

The transdermal delivery (TD) of drugs is nowadays an intense field of research. This technique has several advantages over other drug delivery routes such as the noninvasiveness, the self-administration possibility, the gastrointestinal and liver metabolism avoidance, the continuous and controlled drug administration possibility, and the reduction of side effects. The skin is, however, an effective barrier to external compounds, turning the TD a very challenging process for most drugs.

The soft tissue covering the vertebrates, called skin, is fundamental to their survival on land because of the skin interface role between the environment and the body interior. The skin acts as a barrier between the external hostile environment and the vertebrate's interior. It protects against mechanical injuries, chemicals, pathogens, radiations, and most importantly, against the water and electrolytes lost [1].

The skin has a multilayer structure, with three main layers, namely the epidermis, dermis, and hypodermis (from the skin surface to the interior). The epidermis can be divided into sub-layers or stratum according to the level of keratinization of its cells, and the stratum corneum (SC) is the outermost sublayer. The living keratinocytes of inner epidermis suffer keratinization during the migration to the SC. These cells die, elongate, and become filled with granules, keratin filaments, and with the reminiscent of organelles. The resulting differentiated and dead cells in the SC are named corneocytes and are surrounded by a lipid matrix (LM) [2]. The SC is considered the layer responsible by the barrier properties of the skin, because of its corneocytes organization (tight and without interstices) and keratinization, as well as because of the presence of LM surrounding these cells [3,4].

Contrary to most biological membranes the LM doesn't have phospholipids, instead, it is mainly composed of ceramides (CER), cholesterol (CHOL) and free fatty-acids (FFA), in an equimolar lipid proportion [5-7]. The CER are a complex group of sphingolipids containing sphingosine bases in amide linkage with fatty-acids, existing a great variability of the fatty-acid length on skin CER. The most common CER in LM are the CER NS (nonhydroxy fatty-acid sphingosine CER), also named CER 2, and the CER NP (nonhydroxy fatty-acid phytosphingosine CER), also named CER 3 [8,9]. Most of FFA in the SC are saturated and long, being the lignoceric FFA (with 24 carbons length) the most common [10].

The LM is most of the time represented as stacked lamellar sheets of lipids, with each sheet in a bilayer conformation similar to the cell membranes. There is, however, some debate in the literature about how the CER, CHOL and FFA lipids are packed in the LM. Bouwstra and collaborators suggested that the LM lipids arrange into a trilayer organization, with the inner zone with a higher content of CHOL and FFA, turning that zone more fluid [11]. Later Norlén proposed that the LM is a single gel phase and that the CER can be found in unusual splayed chain conformation, with its two alkyl chains pointing into opposite directions [12]. Recent works applying techniques such as cryo-electron

\footnotetext{
* Corresponding author.

E-mail address: artur@deb.uminho.pt (A. Cavaco-Paulo).
} 


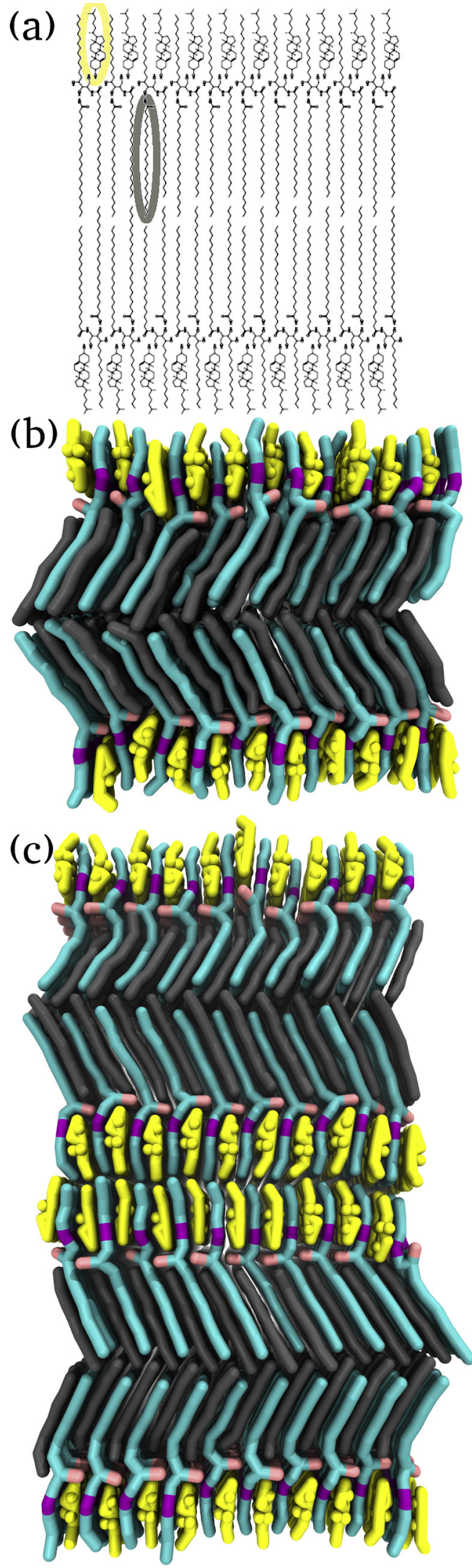

Fig. 1. (a) Iwai LM model [15], with one CHOL and one FFA highlighted in yellow and grey, respectively. Developed LM models, with two ((b) - DOUB model) and four layers ((c) - BIDOUB model). The lipids in the computational models are represented by wide sticks, with the CER in cyan, the CHOL at yellow and the FFA in grey. microscopy and neutron diffraction have been providing new clues in this field [13-15]. Iwai, Norlén, and colleagues proposed in 2012 a new model of the lipid packing in the LM, based on a computational reconstruction of the band patterns presented between corneocytes in cryo-electron microscopy pictures of SC [15]. Contrary to the common representation of LM, the proposed model suggests a lipid assembling with all CER in the splayed conformation. The CHOL molecules are located near the CER sphingoid chains and the FFA near to the fattyacid moieties of the CER (see Fig. 1(a)). This model can explain the barrier properties of the LM since the packing of the lipids is much more thigh and ordered than the packing in the most biological lipid layers.

The SC and LM, as the main players in the skin barrier, are extensively studied for the development of new and more effective strategies for TD of drugs. However, the researchers also have been driving their attention for the drug delivery through hair follicles, because recent works have shown that the potential of this route in TD can be much higher than previously thought [16-18]. Hair follicles only occupy about $0.1 \%$ of the skin surface, however, these structures greatly increase the skin surface area since they correspond to invaginations of the epidermis. The hair follicle ducts are filled with sebum that can influence the transfollicular delivery of drugs, turning important a better understanding of the sebum composition, formation, and properties to increase the TD potential of some drugs.

The sebum is the oily product of the sebaceous glands, which are found in almost all mammals. The main function of human sebum, the metabolic pathways regulating its secretion and composition is not fully understood. There are, however, some suggested functions. The sebum may serve as a delivery system for antioxidants (vitamin E), antimicrobial lipids and pheromones, providing a protective and hydrophobic coating to skin surface and hairs $[19,20]$. The lipids that compose this oily product are the squalene (SQ), FFA, triglycerides (TG), wax esters (WE), CHOL and cholesterol oleate (CHOLO), with median molar percentage values for human samples of 10.6, 28.3, 32.5, 25, 4 and 2, respectively [21]. The sebum also present vestiges of vitamin $\mathrm{E}$ and its composition is characteristic of each species, with the SQ and WE lipids not found elsewhere in mammal's bodies.

Nowadays, most of the research for determining the skin permeability of drugs and to develop better TD strategies are performed through in vitro or ex vivo assays. These experiments are expensive, time-consuming and the use of animal skin for testing has been banned. Molecular dynamics (MD) simulations may serve as an excellent tool to investigate the molecular mechanisms of drug permeation through the skin, overcoming some of these problems. Despite its potential, the number of studies applying MD to improve our knowledge about the skin barrier effect is low. There are, however, some works that study the LM lipids properties and assembling, or the potential of some chemicals to cross the skin barrier. These studies present several different LM models, varying in terms of composition, packing, and complexity [22-25]. However, like in any computational simulation, the ability to correlate the simulation results with the real world depends on the strength and reliability of the computational models.

In this work, we developed coarse-grained MD models of skin SC LM and sebum to unveil insights on the molecular mechanisms of TD as well to obtain robust MD models to use in future assays. The LM model is based on the alternative way of LM packing suggested by Iwai [15], with all CERs in the splayed form. This unusual packing showed high compactness, which may help to explain the singular properties of SC, such as its low permeability. From our knowledge, it is the first time that an LM model with this packing is built at a coarse-grained resolution, and the first time that sebum is investigated using MD.

\section{Materials and methods}

\subsection{Molecular dynamics simulations}

All the simulations were run with the GROMACS 4.0.7 software 
package [26], using the MARTINI force-field [27]. The MARTINI forcefield was developed from the beginning to lipid systems such as our models, has a lower molecular resolution that decreases largely the computational costs, its parametrization is based on the correct modeling of partition free energies of a large number of compounds, and it is been applied in several MD studies with success [27-29]. The MARTINI has some limitations, such as the lack or poor representation of some interactions which depend on atomic properties, and possible problems in potential of mean force (PMF) calculations (for more detailed information check the paper of Marrink and Tieleman [28]).

The simulations boxes were triclinic with periodic boundary conditions in all directions. The time step varied between 20 and $30 \mathrm{fs}$, and the LINCS algorithm was used as bond constraint [30]. When present, the water was modeled with standard MARTINI water beads (beads of P4 type, each one modeling 4 water molecules).

The simulation protocol was similar to all systems, it begins with the energy minimization of the systems for 5000 steps using the steepest descent method, followed by one short run $(1.5 \mathrm{~ns})$ in the isothermalisobaric ensemble (NPT, constant number of molecules, pressure, and temperature) applying positions restraints to all non-solvent beads (with a harmonic force of $10^{3} \mathrm{~kJ} / \mathrm{mol} . \mathrm{nm}^{2}$ ). This preparatory run allows the structures relaxing and the generation of the velocities according to a Maxwell-Boltzmann distribution for the temperature of $306 \mathrm{~K}$. Many MD works about LM use the human body temperature of $310 \mathrm{~K}$ as the reference, but the temperature of skin epidermis is slightly lower, being in the interval of $301 \sim 306 \mathrm{~K}\left(28 \sim 33^{\circ} \mathrm{C}\right)$ [31]. After the minimization and initialization steps, production runs were performed at least in triplicate with a simulation time of at least $600 \mathrm{~ns}$, in an NPT ensemble. Berendsen couplings were used to maintain the temperature of the systems at $306 \mathrm{~K}$ with $0.3 \mathrm{ps}$ as relaxation time, and to maintain the pressure at $1 \mathrm{~atm}$ with $1 \mathrm{ps}$ as the time constant. The pressure coupling was semi-isotropic with the compressibility of $3 \times 10^{-5}$ bar in both directions [32]. The electrostatics and van der Waals interactions were treated with shift potentials, as suggested in the MARTINI website (www.cgmartini.nl), being the electrostatics interactions in the range of $0-1.2 \mathrm{~nm}$ shifted to 0 , while the Lenard-Jones interactions are shifted to 0 in the $0.9-1.2 \mathrm{~nm}$ range.

Some authors apply a scaling factor to the simulations times because the time scales are not well defined in coarse-grained simulations [28]. We did not applied such factor, meaning that the simulations times indicate in this work can be much longer [28].

\subsection{Steered simulations}

Steered simulations were performed to get insights about the permeation potential of the lipidic structures (LM models and sebum). The simulation protocol was the same, with the exception that in the production run one target molecule is forced to cross the lipid structures through the application of a harmonic force of $1000 \mathrm{~kJ} \cdot \mathrm{mol}^{-1} \cdot \mathrm{nm}^{-2}$ to its center of mass during approximately $15 \mathrm{~ns}$. Nile-Red (NRD) and water were the molecules chosen. The resulting trajectories allowed the generation of different configurations along the $\mathrm{z}$ coordinate to perform umbrella sampling simulations [33] and later calculate the potential of mean force (PMF) of the crossing process using free weighted histogram analysis (g_wham tool of GROMACS) [33,34]. Each umbrella configuration was simulated by $20 \mathrm{~ns}$, with the pulled molecule restrained. The position of the restrained molecules differs around $0.1 \mathrm{~nm}$ from the neighboring configurations, resulting in more than 100 configurations sampled. The first three configurations of steered simulations with the developed LM model and NRD or water were removed from the PMF calculation due to the low number of counts in its umbrella histograms. We then adjusted the first PMF value to zero $\mathrm{Kcal} / \mathrm{mol}$ and apply the same adjustment to all remaining PMF values. In the SM are included some umbrella histograms, which points to a good sampling of the pulling process (see Graph S8 in SM).

\subsection{Molecules parametrization}

Different compounds were used in this work, most of them were lipids. Due to its acyl chains and the coarser nature of MARTINI, the parametrization of lipids is very similar between them. All the topologies parameters, except for the CHOL, CHOLO, SQ, polylactic acid (PLA) and NRD were built according to topologies for similar compounds available on the MARTINI website (see the "martini_v2.0_lipids.itp" and "martini_v2.0_surfactants.itp" files, available at http:// www.cgmartini.nl/index.php/60-downloads/forcefield-parameters/ specific-topologies), which generally result from works of the MARTINI developers, and follow the force-field guidelines [35,36].

The CHOL, CHOLO, and triolein (glyceril trioleate, TO) topology parameters were obtained from MARTINI website and are based on Ingólfsson work for CHOL [36], and on Vuorela work for CHOLO and TO [37].

Because the topologies for SQ, NRD and PLA, or similar molecules, were not on MARTINI database we parameterized them applying the protocol described in the force-field webpage for new molecules parameterization using atomistic simulation (http://www.cgmartini.nl/ index.php/tutorials-general-introduction/parametrzining-new-

molecule). We used the Automatic Topology Builder server (atb.uq.edu.au) to obtain atomistic topologies parameters for these molecules (for GROMOS 54A7 force-field) [38,39]. Then the generated atomistic data of in water simulations, mainly the values and forces of the bonds and angles, were used as a reference to coarse-grained parametrization. Note that the PLA was parameterized with 100 monomers of lactic acid.

Finally, the poloxamer (PLX) molecule parametrization follows a different approach. This molecule is a triblock polymer with central hydrophobic monomers of polypropylene oxide (PPO) flanked by hydrophilic monomers of polyethylene oxide (PEO). To model these monomers we add two new particle types to the standard 18 particles in MARTINI, following the methodology described in one work of Hwankyu Lee, in which the PEO monomer topology parameters are studied [40]. The parameters calculated in this work, as well in one posterior work of the same author [41], were used for the new particle representing the PEO monomer. For the PPO particle, the parameters were taken from the work of Hezaveh and colleagues [42]. The PLX polymer in this work has 13 hydrophilic PEO monomers in each tip and 74 hydrophobic PPO monomers in the center. Both PLA and PLX polymers were linear.

It should be noted that the coarse nature of MARTINI, in which one particle generally models 4 heavy atoms, limits the resolution. Two FFA, for example, can differ in just one heavy atom (one carbon) being impossible to represent such difference in the MARTINI resolution. So, exemplifying, when we talk about the palmitic FFA (16 carbons) coarsegrained model we should remember that this model can be a coarser representation of FFAs with 14-18 carbons (16 \pm 2 carbons). More information about the molecules parametrization and its parameters are present in the Supplementary Material (SM) file.

\subsection{Lipid matrix model building}

As stated previously the coarse-grained models of LM built in this work were based on the model suggested by Iwai and collaborators (see Fig. 1(a)) [15]. Also, the lipid composition was the same, with CER NP, CHOL and FFA (lignoceric acid) at 1:1:1 molecular proportion. These lipids and proportions reflect the composition of SC LM [5-7,15]. To obtain such configuration, we initially built 3D conformation files of the three lipids with more straight structures, mainly for the CER and FFA acyl chains, respecting the MARTINI force-field parameters for bond distances. After, we put one CHOL and one FFA molecules models very near to the CER sphingoid and fatty-acid moieties, respectively, with their polar groups aligned with the CER headgroup, as suggested in Iwai work. The resulting three lipids conformation was posteriorly replicated in the $x, y$, and $z$ directions using the GROMACS tool genconf, obtaining 
very ordered layers with the wanted configuration (see Figure S1(a) in $\mathrm{SM})$. Note that when the systems do not contain water, the lipids at the membrane extremities are not facing vacuum but other lipids due to the periodic boundary conditions. There is no free volume in the simulations boxes. Finally, we follow the simulation protocol described previously, to check the stability of the model.

The first simulations performed resulted in MD models very similar to the Iwai model, however some molecules of CHOL and FFA diffuse from its initial position to the opposite CER moiety or presented some disorganization (see Figure S1(b) and (c) in SM). This should be a consequence of the initial low density of the system due to the way how the LM structure was built. The pressure coupling was not fast enough to compact the lipid structure before the escape of some lipids from their initial zone.

Running the simulations at lower temperatures was a good strategy to overcome the initial lipid diffusing issue. We observed that simulations at low temperature resulted in a very ordered lipid packing, similar to Iwai suggested LM model, without diffusing of CHOL or FFA from their initial zones (see Figure S1(d) in SM). To get the final LM models we applied one heating protocol. The simulations began at $50 \mathrm{~K}$ by $600 \mathrm{~ns}$, then three smaller steps of $300 \mathrm{~ns}$ were run, increasing the temperature to 100, 200, 275 and $306 \mathrm{~K}$ respectively. Each of the $300 \mathrm{~ns}$ steps ran at constant temperature, and the beads velocities were reinitialized at each simulation start. The visual inspection and the root mean square deviation (RMSD) of the lipids during the heating process, showed that there is a decorrelation from the initial arrange of the lipid matrix (see Graph S1 in the SM). The ordered arrangement is not necessarily dependent on the protocol with low temperatures since the model without application of the low temperatures protocol is still very ordered and much more similar to the Iwai model [15] and our developed model (see the pictures (b) and (c) in Figure S1 of the Supplementary Material) than other lipid membranes. After the obtaining stable dry LM models, we increase the simulations boxes in the top and the bottom, and fill the free space with waters to obtain hydrated LM models. The developed LM models do not show significant differences between dry and hydrated systems. The resulting MD models of LM shown packing stability and were used in the following work.

Also, the spontaneous packing of these lipids was evaluated, building systems, in vacuum and water, with the same lipidic composition but randomly dispersed in the simulation box. The systems were simulated as usual at $306 \mathrm{~K}$ by $600 \mathrm{~ns}$ (Figure S2 in SM).

Previously, in our group, one coarse-grained model of LM was developed by Nuno Azoia [43]. That model comprises CER NS, CHOL, lignoceric FFA and cholesterol sulfate (CHOLS) with ratio portions based on young normal skin, in a configuration of double bilayers. In the present work, we ran simulations with the Azoia model for comparing its performance with our models.

\subsection{Sebum model building}

The composition of our sebum model is present in Table 1 . There are several works where the composition of sebum was studied [44-46], however, there is some variation on the data that probably results from the difficulty of removing the sebum compounds from the rest of skin compounds such as SC lipids or cell debris.

Some authors have been developing formulations of artificial sebum, to test drug transport properties. In 2006, Stefaniak and Harvey summarized the reported composition of skin surface film liquids (sweat and sebum) as well as artificial sebum formulations, published until that time [21]. More recently, Lu in 2009 and Stefaniak in 2010 developed new artificial sebum formulations based on the 2006 review but with compositions more complex and similar to real human sebum $[47,48]$. Our model composition is based on these works and in one work of Nordstrom and colleagues, in which the lipid composition of follicular casts was studied [49]. We used the most common saturated and unsaturated lipids for WE, TG and FFA, in concentrations according
Table 1

Sebum model mass fraction composition. The tail length corresponds to the number of carbons in the lipids aliphatic chains (except to cholesterol which is not linear), the "x2" and " 33 " indicates the number of equal tails that the lipid has ( 2 and 3, respectively). The cholesterol oleate has a lipid tail of 18 carbons (27 carbons of cholesterol and 18 of the tail).

\begin{tabular}{|c|c|c|c|}
\hline Lipid Types & Lipids & Tail length & $(\mathrm{w} / \mathrm{w} \%)$ \\
\hline & $\begin{array}{l}\text { Squalene } \\
\text { (SQ) }\end{array}$ & $30 \mathrm{C}$ & 10 \\
\hline \multirow{3}{*}{$\begin{array}{l}\text { Free fatty-acids } \\
\text { (FFA) }\end{array}$} & & & 26 \\
\hline & $\begin{array}{l}\text { Palmitic acid } \\
\text { (PA) }\end{array}$ & $16 \mathrm{C}$ & 12 \\
\hline & $\begin{array}{l}\text { Palmitoleic acid } \\
\text { (PO) }\end{array}$ & $16 \mathrm{C}$ & 14 \\
\hline \multirow{3}{*}{$\begin{array}{c}\text { Wax Esters } \\
\text { (WE) }\end{array}$} & & & 26 \\
\hline & $\begin{array}{l}\text { Palmityl Palmitate } \\
\text { (PP) }\end{array}$ & $16 \mathrm{Cx} 2$ & 14 \\
\hline & $\begin{array}{l}\text { Oleyl Oleate } \\
(\mathrm{OO})\end{array}$ & $18 \mathrm{Cx} 2$ & 12 \\
\hline \multirow{3}{*}{$\begin{array}{l}\text { Triglycerides } \\
\text { (TG) }\end{array}$} & & & 32 \\
\hline & $\begin{array}{l}\text { Tripalmitin } \\
\text { (TP) }\end{array}$ & $16 \mathrm{Cx} 3$ & 20 \\
\hline & $\begin{array}{l}\text { Triolein } \\
\text { (TO) }\end{array}$ & $18 \mathrm{Cx} 3$ & 12 \\
\hline \multirow[t]{3}{*}{ Sterols } & & & 6 \\
\hline & $\begin{array}{l}\text { Cholesterol } \\
\text { (CHOL) }\end{array}$ & $27 \mathrm{C}$ & 2 \\
\hline & $\begin{array}{l}\text { Cholesterol Oleate } \\
\text { (CHOLO) }\end{array}$ & $27 C+18 C$ & 4 \\
\hline
\end{tabular}

to these works to have a reliable description of human sebum.

To build the sebum model, we insert randomly the computational models of the lipids, following the mass percentages depicted in Table 1 , in a simulation box that was filled with water molecules. Then the simulations were run by $600 \mathrm{~ns}$ at $306 \mathrm{~K}$ according to the protocol described before. In this way, the sebum lipids aggregated forming a big lipid ball. To have sebum layers, were run simulations with triclinic boxes filled only with the sebum lipids, also for 600 ns. After these simulations, we increase the simulation box in the $\mathrm{Z}$ direction and filled the system with water to obtain sebum layers in an aqueous environment. We simulate these systems by $600 \mathrm{~ns}$ with the same protocol described before. All the sebum simulations were performed in triplicate.

\section{Results and discussion}

\subsection{Lipid matrix and sebum models development}

Fig. 1 shows the schematic representation of Iwai suggested LM packing [15] and our MD models. Two main models were used in this work, the double layer model with 1080 lipids (DOUB, two layers of splayed CER, see Fig. 1(b)) and the bi-double layer model with 2160 lipids (BIDOUB, four layers of splayed CER, see Fig. 1(c)).

It seems that the initial configuration of the lipids is important to obtain ordered models. The imperfections observed in the first developed systems (see Figure S1 in SM) emerged at the very first steps of the simulations but the resulting lipid packing remains very stable. The application of the low temperature overcome the problem of lipid diffusion and disorganization of the first tests, and the following increase of temperatures did not promote lipid destabilization. The difference between the models with or without the application of the heating protocol can be found in Figure S1 at SM. Both DOUB and BIDOUB models present very ordered structures, with the CER fully splayed and showing some tilt in the FFA chains together with the adjacent fattyacid moieties of CER. The tilt appears in all the systems but with some small differences and, interestingly, is different even inside the same model as shown in Fig. 1(c). The simulations did not present significant differences between the DOUB and BIDOUB models, either alone or 


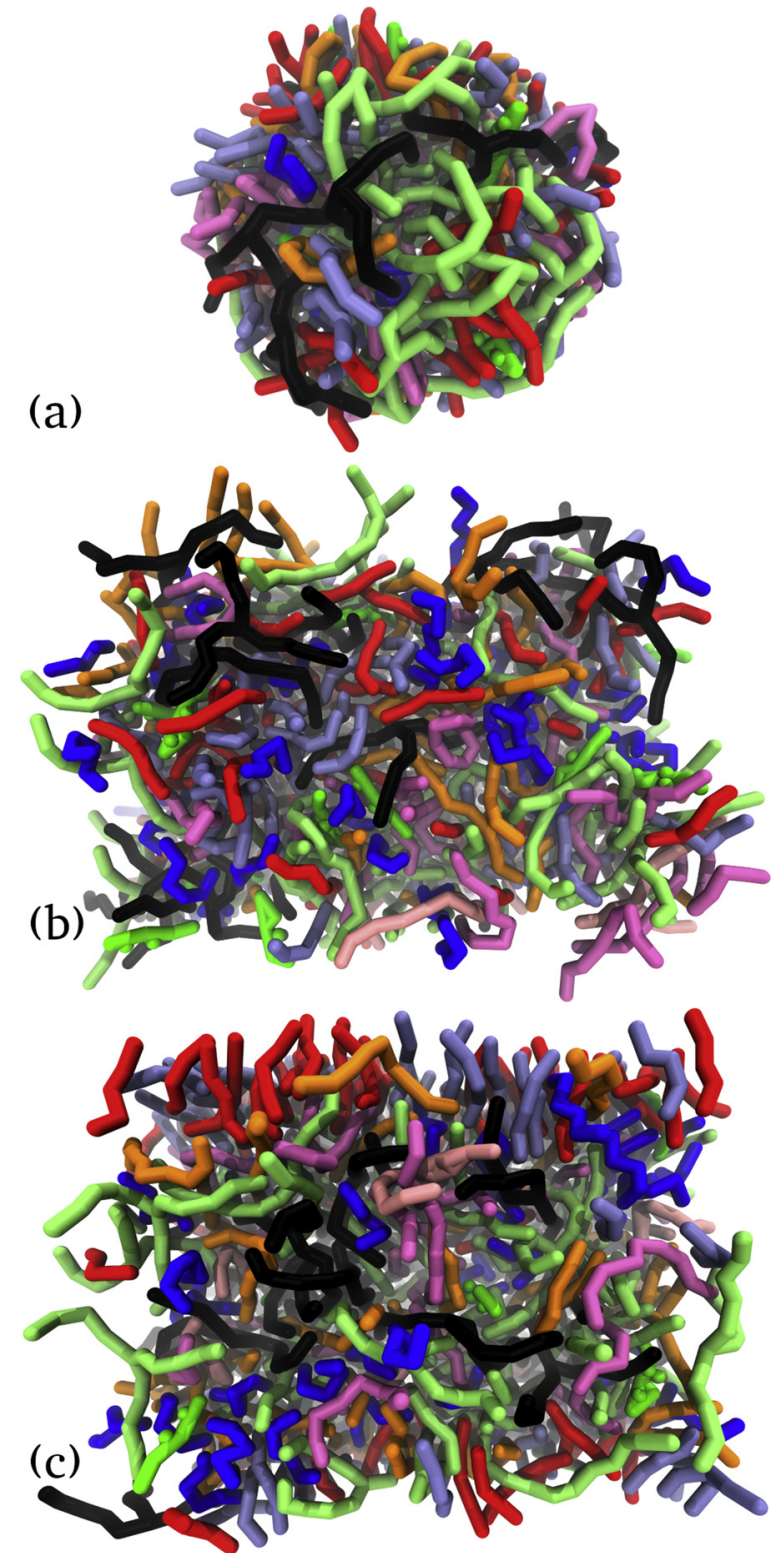

Fig. 2. Configurations after $600 \mathrm{~ns}$ of sebum lipids self-assembling simulations. (a) Sebum lipids in water assembled into a spherical structure. (b) Sebum lipids assembled in the vacuum. (c) The previous system after simulation in water. The water molecules present above and below the lipids, were omitted for clarity. The lipids are depicted as wide sticks, with the following color code: SQ: blue; PA: red; PO: ice-blue; PP: orange; OO: mauve; TP: lime; TO: black; CHOL: green; CHOLO: pink.

interacting with other compounds.

Self-assembling simulations of the LM molecules were also performed. The CER, CHOL, and FFA were inserted randomly and disperse in vacuum or aqueous simulations boxes, with the same number of lipids of previous simulations. The resulting structures were disorganized or mainly in bilayer conformation (in aqueous systems), similar to cell membranes, with the CER in the hairpin configuration and the three lipids equally distributed by the two layers (see Figure S2 in SM). The assembling of the lipids into the arrangement proposed by Iwai [15] should imply a high energy barrier, which can be inaccessible to sample in regular simulations, even at coarse-grained resolutions. The formation and the assembling of lipids in SC LM are processes still not fully understood. Some undescribed mechanisms may facilitate the conformation suggested in Iwai's work and our computational models. One example of these mechanisms was presented in one MD work by
Chinmay Das, which shows that the corneocyte lipid envelop layer surrounding the mammal's corneocytes can induce the formation of layers in the adjacent LM [50].

In Fig. 2, it is presented the sebum in the sphere and layer forms after self-assembly simulations of Table 1 lipids. The resulting structures exhibited much more fluidity than the LM models. When the water is present in the simulated systems, the FFA lipids of the sebum are located more in its surface, with their acids moieties facing the water molecules and their acyl tails more buried in the sebum lipids interior. The simulations also showed that although the sebum lipid clusters formed are fluid, they are stable and there are not lipids diffusing away from the cluster, even briefly, pointing to some viscosity in the model.

\subsection{Interaction simulations}

\subsubsection{Nile-red}

After the achievement of stable LM and sebum models, we perform simulations to study the interaction of these models with some different compounds and between each other. These simulations were performed in aqueous environments, with the surface of the lipids in contact with water beads. One chosen molecule was the NRD. This is a probe widely used in laboratory assays because it is lipophilic and fluorescent, allowing to sense changes in its environment lipophilicity [51,52]. Because of the lipophilic nature of NRD, we expected the insertion of these molecules in the LM interior, however, the NRD was not able to fully insert in the LM model. Just some adsorption at the LM-water interface was visible.

In systems with $10 \mathrm{NRD}$ molecules was observed some aggregation of the NRD in the water-LM interface. We increased the number of these molecules in the simulated systems for 20,100 and 200, testing possible concentration effects. The simulations were also elongated until around 1800 ns. Again the NRD molecules were not able to insert into the LM neither destabilize its lipids, however, the adsorption at the water-lipid interface was more clear, with some NRD forming small grooves on LM surface. In addition, in some replicas with higher concentrations of NRD molecules, emerged zones with the NRD aligned with each other. These observations are depicted in Fig. 3.

The interaction simulations of NRD with sebum showed the expected behavior, namely the full insertion of these lipophilic molecules in the sebum interior as shown in the Fig. 3(c) (whether in spherical and layer sebum forms). Here, the NRD easily inserts in the sebum interior, being located more near to the sebum lipids with oleic acyl chains (except the PO) and to SQ (see the radial distribution function in Graph S2 at SM).

Relatively to our group old LM model, from Azoia's work [43], the simulation results of its interaction with NRD are similar to the sebum, with the full insertion of the NRD molecules in the LM interior, mainly at the middle of the bilayer. In these simulated systems the interaction of NRD is stronger with the CER and FFA molecules of LM (see the radial distribution function in Graph S3 at SM).

The ease insertion of the NRD molecules into the lipid environment of the sebum and old LM model point's to a correct parametrization of this probe (the parametrization is presented in the SM file). The differences observed in the interaction simulations between the three lipid models and the NRD suggests that the alignment of lipids in the developed LM may be too tight, acting as a barrier even to lipophilic molecules such as the NRD. This barrier is entirely physical since the NRD molecules had no problem to insert in the other lipidic structures, which in the case of the old LM model have similar lipidic composition.

\subsubsection{Polylactic acid and poloxamer polymers}

PLA nanoparticles produced through precipitation, using PLX as a surfactant, were developed in our group as drug delivery systems for the skin [53]. These particles presented good stability, efficiency of drug entrapment, and drug release profile, without toxicity for skin 


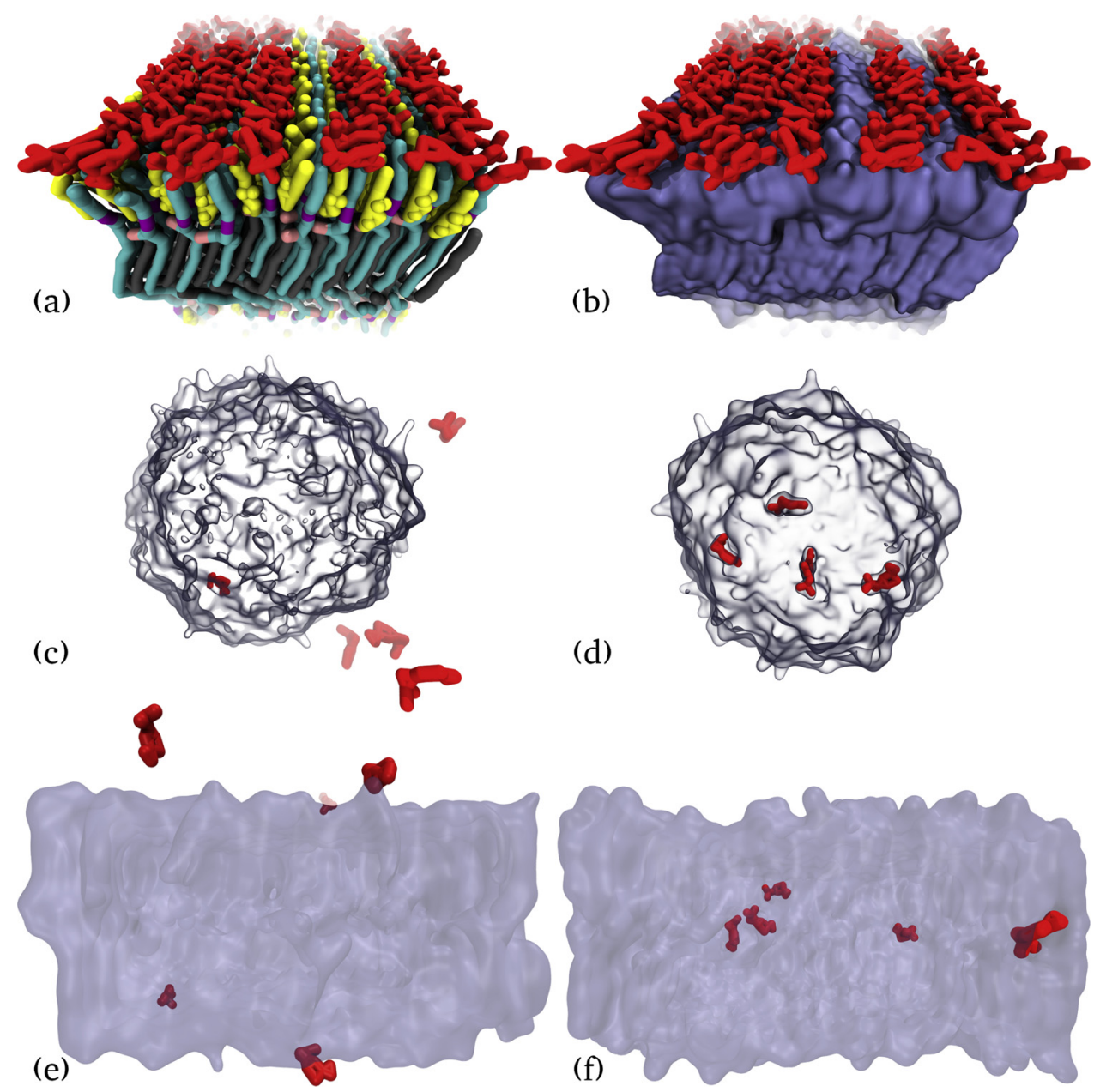

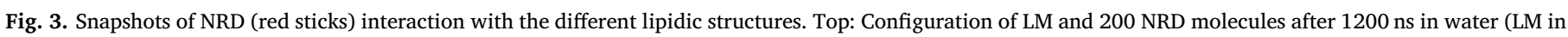

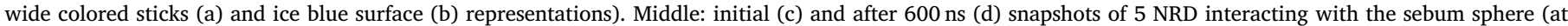

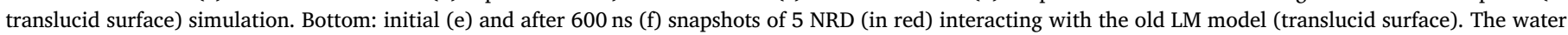
molecules in all systems were excluded for clarity. Note that all the NRD molecules in (c) and (e) snapshots are outside the lipidic structures.

cells. Due to the promising results of this work we performed some simulations of PLA and PLX polymers interaction with the LM, sebum, NRD and between each other.

In all the simulated systems with PLA, no interaction was verified between this molecule and the other molecules, these polymers just aggregate together, and stay in the solvent. This effect is stronger in simulations with acetone as solvent (see Figure S3 in SM).

The PLX presented a different behavior. This polymer is amphipathic, having, in this work, 74 central hydrophobic monomers (PPO monomers) and 26 hydrophilic monomers, 13 in each polymer terminal (PEO monomers). Although its central part is hydrophobic, the aggregation of the PLX in water simulations was weaker than the PLA aggregation at the same conditions. The simulations of NRD interacting with the PLX exhibit the accumulation of NRD molecules in hydrophobic cores formed by some aggregation of the PLX central parts.

The simulations of PLX interaction with sebum and old LM models shown interesting features. When interacting with the old LM model the PLX was able to insert its hydrophobic PPO monomers into the lipidic layer, promoting some destabilization of the LM. The PLX, however, could not insert the same monomers at the sebum model interior, whether the sebum was spherical or layered. In this case, the PLX just adsorbs at the sebum surface, and do not promote changes in the localization of the lipids inside the sebum structure.

Finally, such as in the interaction with NRD, the PLX adsorbs at developed LM surface, with its PPO monomers in more close contact with the lipids and the PEO monomers drifting to the water. The PLX seems to promote a very slight destabilization at the lipid surface, however, is so small that the PLX cannot insert into the LM interior (see Fig. 4).

\subsubsection{Lipid matrix and sebum interaction}

As already stated, the sebum may have a significant effect on the TD of compounds, mainly if the compounds are designed to accumulate into follicular ducts. The interaction simulations between one sphere of sebum and the new LM model showed quick adsorption of the sebum lipids at the LM surface, forming a layer (see Fig. 5). This process just takes around $40 \mathrm{~ns}$ of simulation time. Considering the previous results, in which no compound significantly interacted with this LM model, was surprising to see that the sebum adsorption at the LM surface leads to the diffusion of few CHOL molecules from the LM to the sebum layer (see Fig. 5). This diffusion was quick and did not promote significant structural changes in the LM model. Although we elongate the simulations until $1200 \mathrm{~ns}$, the LM remained stable and no more CHOL drifted from their initial zones at LM structure.

In the case of the older LM model, the simulation of its interaction with sebum sphere was completely different. When the sebum sphere found the old LM model the two lipid structures quickly begin to merge (see Fig. 5 and Video 1 in SM). The sebum lipids are fully absorbed by the LM and all the lipids rearrange. The full absorption process takes less than $600 \mathrm{~ns}$.

Tascini and colleagues have two MD works in which they use molecules of tri-cis-6-hexadecenoin TG as a simple computational model of 


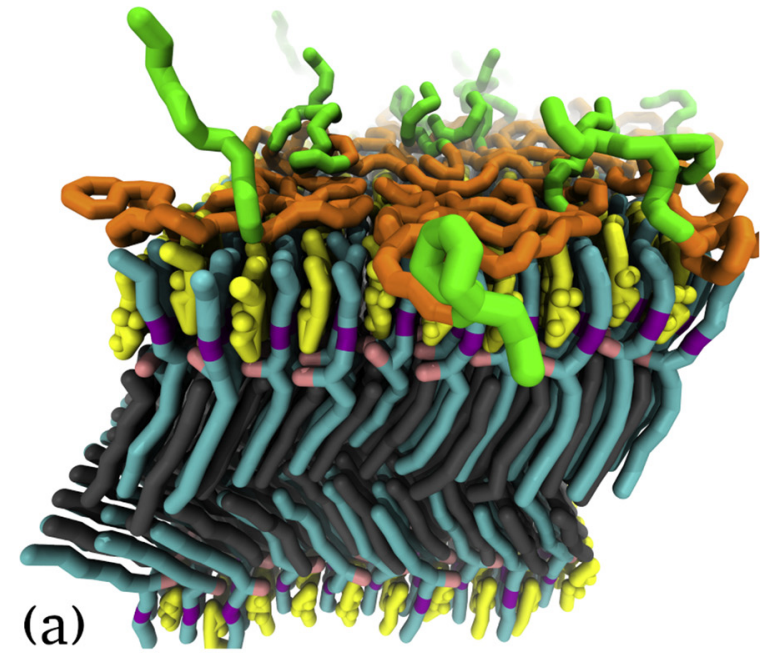

(b)
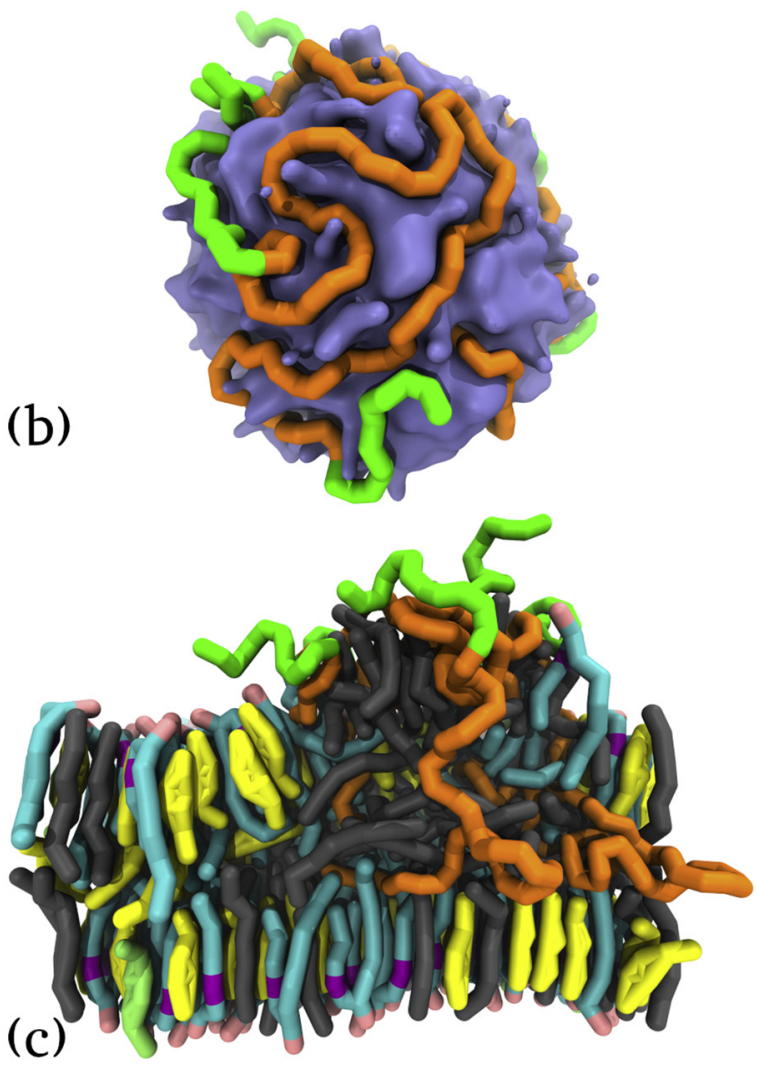

Fig. 4. Lipidic systems snapshots after $600 \mathrm{~ns}$ of interaction simulations with PLX polymers in water. (a) Developed LM model. (b) Sebum model. (c) Old LM model. The developed and old LM models were represented by wide sticks, with CER at cyan (with head groups at pink), CHOL at yellow, CHOLS at lime (only in the old model) and FFA at gray. The sebum sphere is represented as an ice blue surface. The PEO monomers of PLX (hydrophilic) are depicted as green sticks while the PPO (hydrophobic) are orange. The water was omitted for clarity.

sebum oil [54,55]. In their most recent publication [55] they studied the interaction between the TG molecules and SC lipids (CER, CHOL, and FFA). That interaction resulted in some SC lipids escaping from its layer and diffusing to the TG bulk, and some TG were able to penetrate its chains in the SC layer interior.

In our simulations, only CHOL were able to leave the LM, probably because the FFA are buried in the LM interior, not interacting directly with the sebum, and because of the compactness of the LM model that also did not allow the entrance in its interior of the sebum molecules.
Tascini and co-authors pointed out that the escape of the lipids can result of strong interactions of them with the glyceryl groups of TG [55], however, the RDF analysis of our simulations shows that the TG in our sebum model (TO and TP) are not the molecules of the sebum with strongest interactions with the CHOL presented in the LM (see Graph S4 at SM).

The interaction between the sebum and some molecules will certainly play an important role in the TD potential of these molecules. Also, the impact of the sebum in the LM should influence the TD of the drugs. The differences between the simulation findings in the discussed systems (developed LM, old LM, and the Tascini work) shows that more studies are needed to better understand the effect of the sebum in the drugs and skin components, and how these interactions can influence the TD process.

\subsection{Steered simulations}

Sampling some states in standard simulations can be hard or even impossible due to the existence of high free energy barriers, or because these states may require very long simulation times. We applied steered MD simulations, in which molecules of NRD or water were forced to cross the lipid structures, increasing the sampling and allowing the calculation of the potential of mean force (PMF) to obtain insights about the permeation potential of the new LM model.

Unfortunately, we had problems with the steered simulation with the new LM model. The simulations in which the NRD molecule or water bead were forced to cross the LM crashed always before the full crossing of the LM, more precisely when the molecules are trying to cross the CER head-group zone by the second time. It is known that the MARTINI, and other coarse-grained force-fields, have some limitations concerning the calculation of free energies, enthalpies, and entropies [28]. However, it is described that the MARTINI can provide reliable results, mainly if the steered simulations are with hydrophobic solvents or with lipid membranes, and the analysis is qualitative, like in this work [28]. Although the crashes, and the MARTINI limitations on this type of studies, we included the PMF results in this work for several reasons, namely:

- the crashes happen only in the pulling simulations, which objective is to provide the spatial sampling of the process;

- the PMF calculation is based on several small simulations without any problems;

- the system is lipidic and we focus on a qualitative analysis of the PMF results.

Some snapshots of the developed LM model steered simulations, with one NRD molecule, are shown in Fig. 6 (see Figures S4 and S5 in SM for the steered simulations with old LM and sebum models, videos for all steered simulations with NRD are also presented in SM). The calculated PMF for the replicas with intermediate values are also presented in Fig. 6 (see the PMF of all replicas in Graph S5, S6, and S7 in $\mathrm{SM})$. The visual inspection of the steered simulations with NRD denoted some difficulty of this molecule to inserts into the middle of CER and CHOL lipids. Cross the zone with the CER and FFA head groups seems even harder. The PMF variation confirms these observations (see Fig. 6(b)), it begins with some oscillation and a posterior significant decrease. The oscillation should result from the process of the NRD insertion, which leads to the posterior decrease due to the lipophilic environment reached by the NRD. Later, the PMF greatly increases when the NRD is crossing the CER and FFA head groups. A new decrease is seen later until the NRD reaches the more fluid zone of the LM center ( $\mathrm{z}$ around 1.2). Then the PMF increases again as expected (the PMF profile should be symmetric from this point, due to the symmetry of the LM membrane), and ends prematurely due to the simulation crash.

The PMF of NRD crossing the LM model also points to a tight 


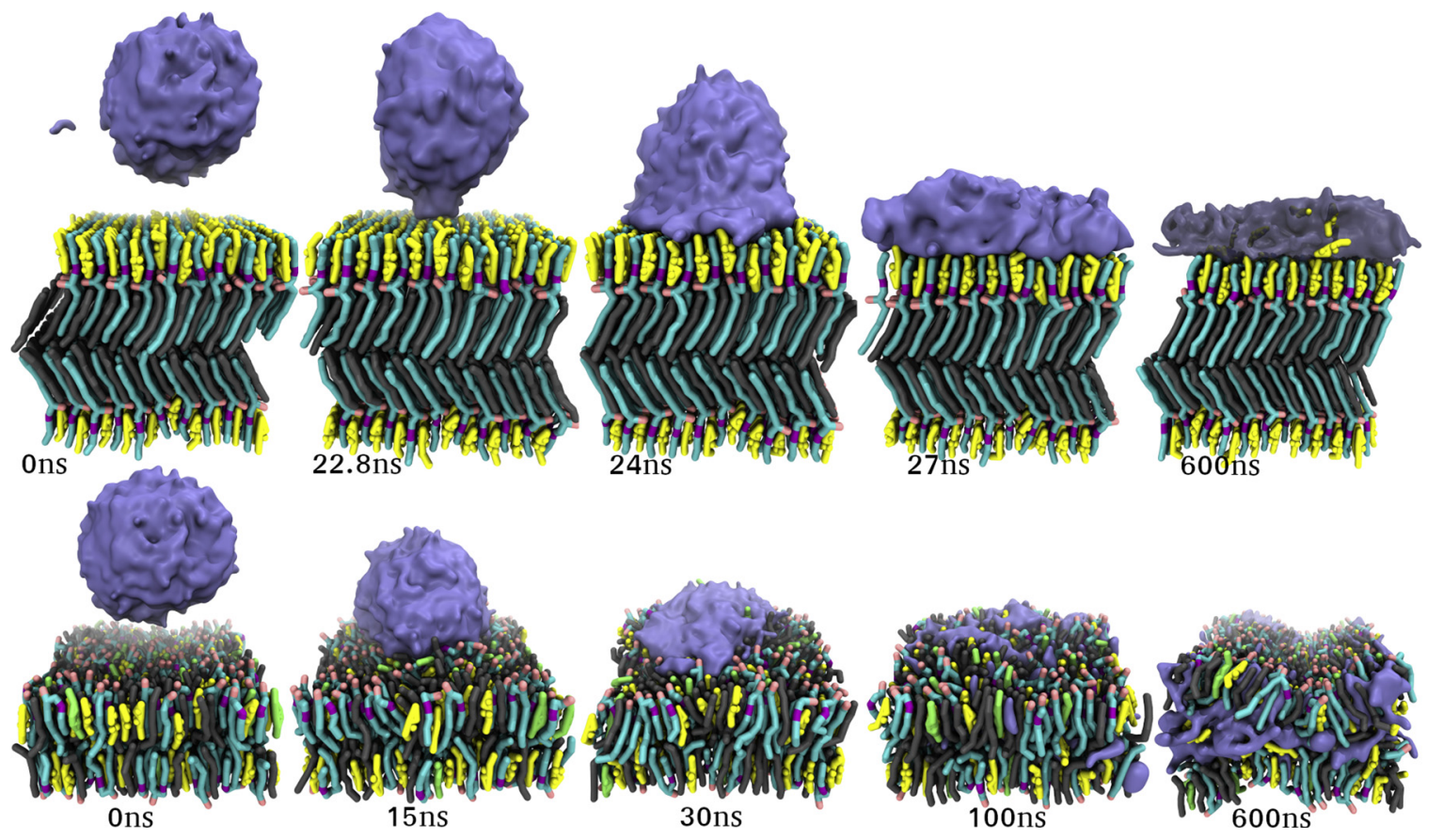

Fig. 5. Time evolution snapshots of the interaction simulations between the sebum and the developed LM model (top), and old LM model (bottom). The LM is represented as sticks and the sebum as surface. The sebum is translucid in the $600 \mathrm{~ns}$ top snapshot to reveal the CHOL molecules drifted from the LM to the sebum interior (yellow sticks). The color code is the same from previous figures. The water is omitted.

organization of the lipids. The increase of energy when the NRD is crossing the CER and FFA head groups is expected since the molecule will probe a more polar environment. However, the increase is big and is visible in the simulations that the NRD had difficulty to cross this zone because of its compactness. The fact that the PMF profile reaches a local minimum when the NRD is in the middle of the LM, which is more fluid, also agrees to the suggestion that the physical arrangement of the lipids in this model is contributing greatly to the PMF profile.

We expected a very different profile for the PMF variation of the water crossing the new LM model, as well as higher values compared with the NRD profile since the NRD is a hydrophobic molecule.
Surprisingly, the PMF profile for the water molecule was similar to the $\mathrm{NRD}$ and presented lightly inferior values of energy. The major difference was that the PMF increases when the water is inserted into the LM interior, while the PMF for the NRD case decreased. After this, the PMF reaches similar values and also presented a local minimum at the middle of LM. This is one more evidence that the major influencer of the PMFs profiles for the developed LM model is the lipids compactness since the chemical properties such as the hydrophobicity and polarity have a small impact on the computed energies, being overcome by the physical resistance of the lipids to the crossing of the molecules. The smaller values of PMF for the water molecule should result from its

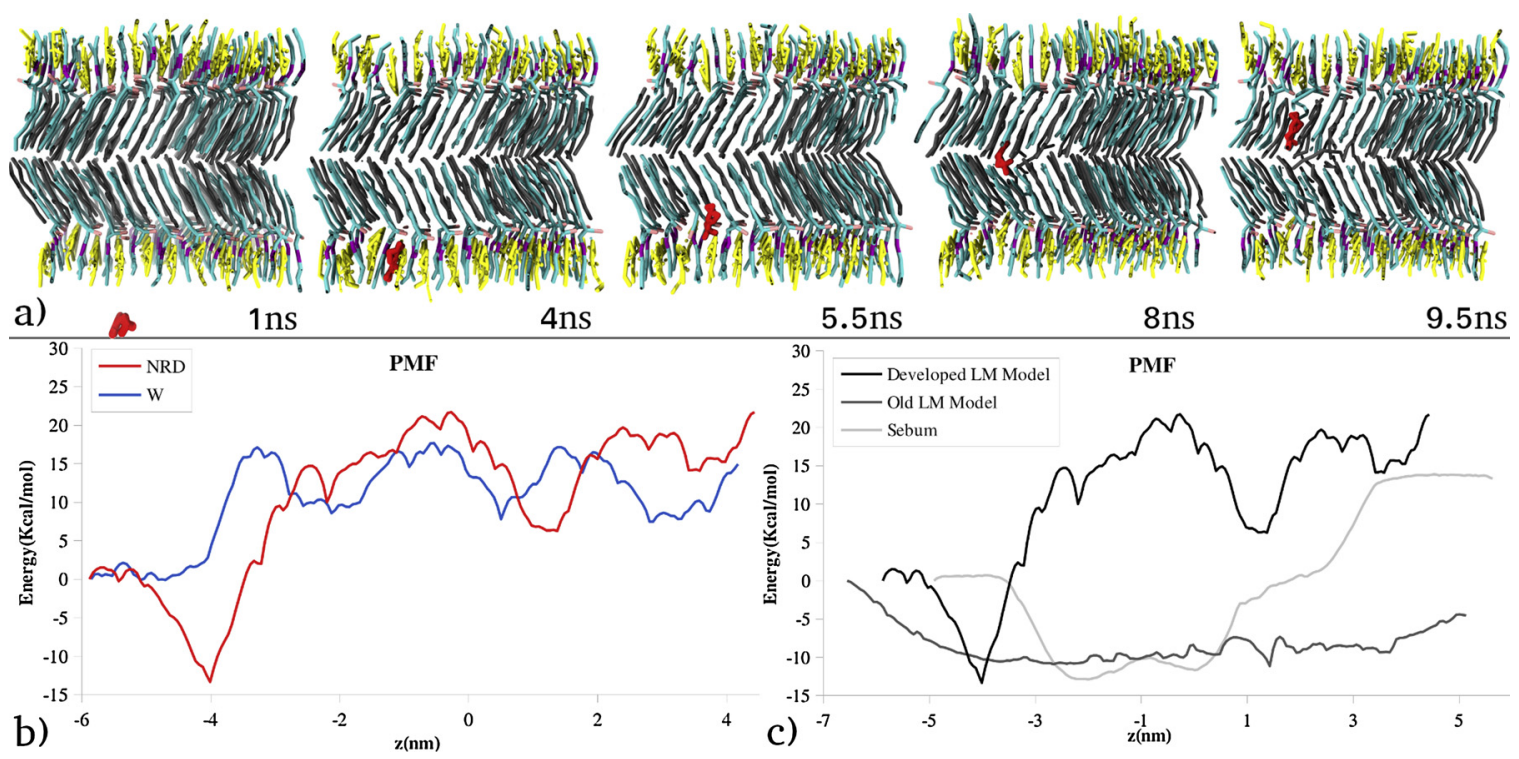

Fig. 6. (a) Snapshots of steered simulation evolution with one NRD pulled through the LM model. The same representation type of the previous figures was used. (b) Calculated PMF variation for the steered simulation of NRD (red line), and water (blue line) crossing the LM model. (c) Calculated PMF variation for NRD pulling simulations through the LM model (black line), the old LM model (gray line), and the sebum model (blue line). The $\mathrm{z}$ is relative to the center of the simulation box. The middle of LM is around $1 \mathrm{~nm}$. 
smaller size (one coarse-grained bead for water against eleven for NRD).

The PMF variation for the systems with NRD crossing the older LM model and the sebum model presented more expectable profiles (see Fig. 6(c)). There is the decrease of free energy when the NRD inserts into the lipid interior, with the posterior increase when the molecule leaves the lipids in the opposite side. It was interesting to note that the decrease of PMF when the NRD inserts on the lipid interior was similar for the three lipid systems, namely around $10 \sim 12 \mathrm{Kcal} / \mathrm{mol}$.

Magnus Lundborg and collaborators published recently two extensive works in which they also developed an LM model based on Iwai work, although the final lipid composition and configuration were changed [56,57]. Their model also presented stability with the splayed CER and some tilt in the zone where the FFA are located, remarks that are also presented in one recent work of neutron diffraction of skin lipids [58]. These results agree with the performed tests in our work, however, the PMF profiles in the Lundborg's work were different, which could result from more precise calculations, since the model was not coarse-grained, or due to less compactness in the LM model. It should be noted that some authors propose models of LM packing in which there are zones with different phases, co-existing gel/crystalline phases with fluid/liquid phases [11,59]. The lipid packing configuration proposed by Iwai [15] and studied in this work can correspond to more crystalline and impermeable zones. This issue of LM variability should be a target of more intense research to allow the improvement of computational or even in vitro and ex vivo models of skin SC models.

\section{Conclusions}

In this work, MD models of LM and sebum were developed and their interaction with some compounds was tested. From our knowledge is the first time that one MD model of sebum is developed, and the first time that one coarse-grained LM model with full splayed CER is developed.

The simulations showed that this unusual packing is stable, promotes compactness, has low interaction potential with other compounds, and low permeability.

The simulations also showed a strong interaction between the sebum and the LM models, pointing to a significant impact of the sebum in the TD of compounds. In addition, the sebum model can allow the study of how the drugs can behave at follicular ducts that are filled with this oil.

The two developed models showed stability, robustness, and can be used in other works to study the mechanisms of TD, providing unique information about how drugs can interact with the sebum and LM at the molecular level.

\section{Author contributions}

The manuscript was written through the contribution of all authors. All authors approved the final version of the manuscript.

\section{CRediT authorship contribution statement}

Egipto Antunes: Conceptualization, Methodology, Funding acquisition, Data curation, Investigation, Writing - original draft, Writing review \& editing. Artur Cavaco-Paulo: Supervision, Writing - review \& editing, Funding acquisition.

\section{Declaration of Competing Interest}

The authors declare that they have no known competing financial interests or personal relationships that could have appeared to influence the work reported in this paper.

\section{Acknowledgments}

We thank the Portuguese Foundation for Science and Technology (FCT) for providing the grant for part of Egipto Antunes Ph.D. studies (scholarship SFRH/BD/122952/2016), and to also support this work under the scope of the strategic funding of UID/BIO/04469/2019 unit and BioTecNorte operation (NORTE-01-0145-FEDER-000004) funded by the European Regional Development Fund under the scope of Norte2020 - Programa Operacional Regional do Norte. We want to thank the access of Minho University "SeARCH" ("Services and Advanced Research Computing with HTC/HPC clusters") cluster. We also thank Tarsila Castro for its technical advice and text revision.

\section{Appendix A. Supplementary data}

Supplementary material related to this article can be found, in the online version, at doi:https://doi.org/10.1016/j.colsurfb.2020.110928.

\section{References}

[1] K.R. Feingold, Thematic review series: skin lipids. The role of epidermal lipids in cutaneous permeability barrier homeostasis, J. Lipid Res. 48 (2007) 2531-2546.

[2] R.K. Freinkel, D.T. Woodley, The Biology of the Skin, CRC Press, London, 2001.

[3] D.N. Menton, A minimum-surface mechanism to account for the organization of cells into columns in the mammalian epidermis, Am. J. Anat. 145 (1976) 1-22.

[4] P.W. Wertz, B. van den Bergh, The physical, chemical and functional properties of lipids in the skin and other biological barriers, Chem. Phys. Lipids 91 (1998) 85-96.

[5] L. Norlén, I. Nicander, B. Lundh Rozell, S. Ollmar, B. Forslind, Inter- and intraindividual differences in human stratum corneum lipid content related to physical parameters of skin barrier function in vivo, J. Invest. Dermatol. 112 (1999) 72-77.

[6] A. Weerheim, M. Ponec, Determination of stratum corneum lipid profile by tape stripping in combination with high-performance thin-layer chromatography, Arch. Dermatol. Res. 293 (2001) 191-199.

[7] W. Philip, N. Lars, Skin, hair, and nails: structure and function, in: B. Forslind, M. Lindberg (Eds.), Ski. Hair, Nails Struct. Funct. CRC Press, New York, 2003.

[8] M. Rabionet, K. Gorgas, R. Sandhoff, Ceramide synthesis in the epidermis, Biochim. Biophys. Acta 1841 (2014) 422-434.

[9] H. Farwanah, K. Raith, R.H.H. Neubert, J. Wohlrab, Ceramide profiles of the uninvolved skin in atopic dermatitis and psoriasis are comparable to those of healthy skin, Arch. Dermatol. Res. 296 (2005) 514-521.

[10] L. Norlén, I. Nicander, A. Lundsjö, T. Cronholm, B. Forslind, A new HPLC-based method for the quantitative analysis of inner stratum corneum lipids with special reference to the free fatty acid fraction, Arch. Dermatol. Res. 290 (1998) 508-516.

[11] J.A. Bouwstra, F.E. Dubbelaar, G.S. Gooris, M. Ponec, The lipid organisation in the skin barrier, Acta Derm. Venereol. Suppl. (Stockh) 208 (2000) 23-30.

[12] L. Norlén, Skin barrier structure and function: the single gel phase model, J. Invest. Dermatol. 117 (2001) 830-836.

[13] A. Al-Amoudi, J. Dubochet, L. Norlén, Nanostructure of the epidermal extracellular space as observed by cryo-electron microscopy of vitreous sections of human skin, J. Invest. Dermatol. 124 (2005) 764-777.

[14] E.H. Mojumdar, D. Groen, G.S. Gooris, D.J. Barlow, M.J. Lawrence, B. Deme, J.A. Bouwstra, Localization of cholesterol and fatty acid in a model lipid membrane: a neutron diffraction approach, Biophys. J. 105 (2013) 911-918.

[15] I. Iwai, H. Han, L. Den Hollander, S. Svensson, L.-G. Öfverstedt, J. Anwar, J. Brewer, M. Bloksgaard, A. Laloeuf, D. Nosek, S. Masich, L. a Bagatolli, U. Skoglund, L. Norlén, The human skin barrier is organized as stacked bilayers of fully extended ceramides with cholesterol molecules associated with the ceramide sphingoid moiety, J. Invest. Dermatol. 132 (2012) 2215-2225.

[16] Y.Y. Grams, L. Whitehead, P. Cornwell, J.A. Bouwstra, Time and depth resolved visualisation of the diffusion of a lipophilic dye into the hair follicle of fresh unfixed human scalp skin, J. Control. Release 98 (2004) 367-378.

[17] J. Lademann, N. Otberg, H. Richter, H.J. Weigmann, U. Lindemann, H. Schaefer, W. Sterry, Investigation of follicular penetration of topically applied substances, Skin Pharmacol. Appl. Skin Physiol. 14 (Suppl 1) (2001) 17-22.

[18] E.A. Essa, M.C. Bonner, B.W. Barry, Human skin sandwich for assessing shunt route penetration during passive and iontophoretic drug and liposome delivery, J. Pharm. Pharmacol. 54 (2002) 1481-1490.

[19] T. Nikkari, Comparative chemistry of sebum, J. Invest. Dermatol. 62 (1974) $257-267$

[20] K.R. Smith, D.M. Thiboutot, Thematic review series: skin Lipids. Sebaceous gland lipids: friend or foe? J. Lipid Res. 49 (2008) 271-281.

[21] A.B. Stefaniak, C.J. Harvey, Dissolution of materials in artificial skin surface film liquids, Toxicol. Vitr. 20 (2006) 1265-1283.

[22] R. Gupta, B. Rai, Molecular dynamics simulation study of skin lipids: effects of the molar ratio of individual components over a wide temperature range, J. Phys. Chem. B 119 (2015) 11643-11655.

[23] T.C. Moore, R. Hartkamp, C.R. Iacovella, A.L. Bunge, C. McCabe, Effect of ceramide tail length on the structure of model stratum corneum lipid bilayers, Biophys. J. 114 (2018) 113-125.

[24] C. Das, P.D. Olmsted, The physics of stratum corneum lipid membranes, Philos. 
Trans. R. Soc. A Math. Phys. Eng. Sci. 374 (2016) 20150126.

[25] R. Notman, J. Anwar, Breaching the skin barrier - Insights from molecular simulation of model membranes, Adv. Drug Deliv. Rev. 65 (2013) 237-250.

[26] B. Hess, C. Kutzner, D. van der Spoel, E. Lindahl, GROMACS 4: algorithms for highly efficient, load-balanced, and scalable molecular simulation, J. Chem. Theory Comput. 4 (2008) 435-447.

[27] S.J. Marrink, H.J. Risselada, S. Yefimov, D.P. Tieleman, A.H. de Vries, The MARTINI force field: coarse grained model for biomolecular simulations, J. Phys. Chem. B 111 (2007) 7812-7824.

[28] S.J. Marrink, D.P. Tieleman, Perspective on the martini model, Chem. Soc. Rev. 42 (2013) 6801-6822.

[29] H.I. Ingólfsson, Ca. Lopez, J.J. Uusitalo, D.H. de Jong, S.M. Gopal, X. Periole, S.J. Marrink, The power of coarse graining in biomolecular simulations, Wiley Interdiscip. Rev. Comput. Mol. Sci. 4 (2014) 225-248.

[30] B. Hess, H. Bekker, H.J.C. Berendsen, J.G.E.M. Fraaije, LINCS: a linear constraint solver for molecular simulations, J. Comput. Chem. 18 (1997) 1463-1472.

[31] I. Plasencia, L. Norlén, L.A. Bagatolli, Direct visualization of lipid domains in human skin stratum corneum's lipid membranes: effect of $\mathrm{pH}$ and temperature, Biophys. J. 93 (2007) 3142-3155.

[32] H.J.C. Berendsen, J.P.M. Postma, W.F. van Gunsteren, A. DiNola, J.R. Haak, Molecular dynamics with coupling to an external bath, J. Chem. Phys. 81 (1984) 3684.

[33] B. Roux, The calculation of the potential of mean force using computer simulations, Comput. Phys. Commun. 91 (1995) 275-282.

[34] J.S. Hub, B.L. de Groot, D. van der Spoel, g_wham—a free weighted histogram analysis implementation including robust error and autocorrelation estimates, J. Chem. Theory Comput. 6 (2010) 3713-3720.

[35] T.A. Wassenaar, H.I. Ingólfsson, R.A. Böckmann, D.P. Tieleman, S.J. Marrink, Computational lipidomics with insane : a versatile tool for generating custom membranes for molecular simulations, J. Chem. Theory Comput. 11 (2015) $2144-2155$.

[36] H.I. Ingólfsson, M.N. Melo, F.J. van Eerden, C. Arnarez, C. a Lopez, T. a Wassenaar, X. Periole, A.H. de Vries, D.P. Tieleman, S.J. Marrink, Lipid organization of the plasma membrane, J. Am. Chem. Soc. 136 (2014) 14554-14559.

[37] T. Vuorela, A. Catte, P.S. Niemelä, A. Hall, M.T. Hyvönen, S.J. Marrink, M. Karttunen, I. Vattulainen, Role of lipids in spheroidal high density lipoproteins, PLoS Comput. Biol. 6 (2010) e1000964.

[38] A.K. Malde, L. Zuo, M. Breeze, M. Stroet, D. Poger, P.C. Nair, C. Oostenbrink, A.E. Mark, An automated force field topology builder (ATB) and repository: version 1.0, J. Chem. Theory Comput. 7 (2011) 4026-4037.

[39] N. Schmid, A.P. Eichenberger, A. Choutko, S. Riniker, M. Winger, A.E. Mark, W.F. van Gunsteren, Definition and testing of the GROMOS force-field versions 54A7 and 54B7, Eur. Biophys. J. 40 (2011) 843-856.

[40] H. Lee, A.H. De Vries, S.J. Marrink, R.W. Pastor, A coarse-grained model for polyethylene oxide and polyethylene glycol: conformation and hydrodynamics, J. Phys. Chem. B 113 (2009) 13186-13194.

[41] H. Lee, R.W. Pastor, Coarse-grained model for pegylated lipids: effect of pegylation on the size and shape of self-assembled structures, J. Phys. Chem. B 115 (2011) 7830-7837.

[42] S. Hezaveh, S. Samanta, A. De Nicola, G. Milano, D. Roccatano, Understanding the interaction of block copolymers with DMPC lipid bilayer using coarse-grained molecular dynamics simulations, J. Phys. Chem. B 116 (2012) 14333-14345.

[43] M. Martins, N.G. Azoia, A. Ribeiro, U. Shimanovich, C. Silva, A. Cavaco-Paulo, In vitro and computational studies of transdermal perfusion of nanoformulations containing a large molecular weight protein, Colloids Surf. B Biointerfaces 108 (2013) 271-278.

[44] A. Pappas, S. Johnsen, J.-C. Liu, M. Eisinger, Sebum analysis of individuals with and without acne, Dermatoendocrinology 1 (2009) 157-161.

[45] M. Picardo, M. Ottaviani, E. Camera, A. Mastrofrancesco, Sebaceous gland lipids, Dermatoendocrinology 1 (2009) 68-71.

[46] L.C. Robosky, K. Wade, D. Woolson, J.D. Baker, M.L. Manning, D. a Gage, M.D. Reily, Quantitative evaluation of sebum lipid components with nuclear magnetic resonance, J. Lipid Res. 49 (2008) 686-692.

[47] G.W. Lu, S. Valiveti, J. Spence, C. Zhuang, L. Robosky, K. Wade, A. Love, L.-Y. Hu, D. Pole, M. Mollan, Comparison of artificial sebum with human and hamster sebum samples, Int. J. Pharm. 367 (2009) 37-43.

[48] A.B. Stefaniak, C.J. Harvey, P.W. Wertz, Formulation and stability of a novel artificial sebum under conditions of storage and use, Int. J. Cosmet. Sci. 32 (2010) $347-355$

[49] K.M. Nordstrom, J.N. Labows, K.J. McGinley, J.J. Leyden, Characterization of wax esters, triglycerides, and free fatty acids of follicular casts, J. Invest. Dermatol. 86 (1986) 700-705.

[50] C. Das, M.G. Noro, P.D. Olmsted, Lamellar and inverse micellar structures of skin lipids: effect of templating, Phys. Rev. Lett. 111 (2013) 148101.

[51] P. Greenspan, S.D. Fowler, Spectrofluorometric studies of the lipid probe, nile red, J. Lipid Res. 26 (1985) 781-789.

[52] M.Ca. Stuart, J.C. van de Pas, J.B.F.N. Engberts, The use of Nile Red to monitor the aggregation behavior in ternary surfactant-water-organic solvent systems, J. Phys Org. Chem. 18 (2005) 929-934.

[53] B. Fernandes, R. Silva, A. Ribeiro, T. Matamá, A.C. Gomes, A.M. Cavaco-Paulo, D. Improved Poly, L-lactide) nanoparticles-based formulation for hair follicle targeting, Int. J. Cosmet. Sci. 37 (2015) 282-290.

[54] A.S. Tascini, M.G. Noro, R. Chen, J.M. Seddon, F. Bresme, Understanding the interactions between sebum triglycerides and water: a molecular dynamics simulation study, Phys. Chem. Chem. Phys. 20 (2018) 1848-1860, https://doi.org/10.1039/ C7CP06889A.

[55] A.S. Tascini, M.G. Noro, J.M. Seddon, R. Chen, F. Bresme, Mechanisms of lipid extraction from skin lipid bilayers by sebum triglycerides, Phys. Chem. Chem. Phys. 21 (2019) 1471-1477.

[56] M. Lundborg, A. Narangifard, C.L. Wennberg, E. Lindahl, B. Daneholt, L. Norlén, Human skin barrier structure and function analyzed by cryo-EM and molecular dynamics simulation, J. Struct. Biol. 203 (2018) 149-161.

[57] M. Lundborg, C.L. Wennberg, A. Narangifard, E. Lindahl, L. Norlén, Predicting drug permeability through skin using molecular dynamics simulation, J. Control. Release (2018) 1-6.

[58] A. Schroeter, S. Stahlberg, B. Školová, S. Sonnenberger, A. Eichner, D. Huster, K. Vávrová, T. Hauß, B. Dobner, R.H.H. Neubert, A. Vogel, Phase separation in ceramide[NP] containing lipid model membranes: neutron diffraction and solidstate NMR, Soft Matter 13 (2017) 2107-2119.

[59] B. Forslind, A domain mosaic model of the skin barrier, Acta Derm. Venereol. 74 (1994) 1-6. 